\title{
Design of Adaptive Fuzzy Sliding Mode for Nonlinear System Control
}

\author{
Sinn-Cheng Lin and Yung-Yaw Chen \\ Department of Electrical Engineering, National Taiwan University, \\ Taipei, Taiwan, R.O.C.
}

\begin{abstract}
An adaptive fuzzy sliding mode controller (AFSMC) is proposed in this paper. The parameters of the membership functions in the fuzzy rule base are changed according to some adaptive algorithm for the purpose of controlling the system state to hit an userdefined sliding surface and then slide along it. The initial IF-THEN rules in the AFSMC can be randomly selected or roughly given by human experts, and then automatically tuned by a direct adaptive law. Therefore, reducing the expertise dependency in the design procedure of fuzzy logic control, we call it rule tolerance property. By applying the AFSMC to control a nonlinear unstable inverted pendulum system, the simulation results show the expected approximation sliding property was occurred, and the dynamic behavior of control system can be determined by the sliding surface.
\end{abstract}

\section{Introduction}

In the classical control theory, most of the automatic control problem are usually solved by mathematical tools based on the system models. But in the real world, there are many complex industrial processes whose available models can't be easily developed. Hence, design a model-based controller to control these ill-defined systems is very difficult. However, human operators can do well without keeping any mathematical model in mind. Fuzzy logic controller (FLC) [1]. Although there has been some successful applications of fuzzy logic controller [2]-[3], however, it still has some problems in the design of FLC: 1) The fuzzy control rules are experience oriented and the suitable membership functions should be given by time-consuming trial and error procedure; 2) The dynamic behavior of control system can't be specified previously; 3) No general stability analysis tools can be applied to a fuzzy system.

To overcome 1) and 2), we had proposed a fuzzy sliding mode control (FSMC) scheme [4] which has the advantages of FLC and SMC (sliding mode control) [5]. By introducing the sliding mode to the FLC and fuzzifying the sliding surface, the FSMC has a fuzzy sliding mode which is similar to crisp sliding mode but has less chattering then the crisp one. The closed loop system can be characterized by a user-defined sliding surface, and the fuzzy rules can be easily obtained. But the FSMC developed in our previous works has no autotunning capacity. The slightly tuning by designer is also necessary. In the paper, we develop a new strategy called adaptive fuzzy sliding mode control (AFSMC). The parameters of the membership functions in the AFSMC are changed according to some adaptive algorithm for the purpose of controlling the system states to hit an user-defined sliding surface and then slide along it. The initial IFTHEN rules in the AFSMC can be randomly selected or roughly given by human experts, and then automatically tuned by a direct adaptive law. Therefore, reducing the expertise dependency in the design procedure of fuzzy logic control, we call it rule tolerance property. In Section 2, we introduce the basic concept of FSMC. The adaptive algorithms for FSMC are derived in Section 3. In Section 4, the AFSMC is applied to control an inverted pendulum system. Section 5 gives a conclusion.

\section{Basic Concept of FSMC}

There are two parts in this section. First, we discuss some definitions of fuzzy logic control. 
Secondly, conventional sliding mode control is briefly reviewed, and then the main idea of fuzzy sling mode control is presented.

\section{A. Some definitions of fuzzy logic control}

In this paper, the membership functions we adopted are all in the form of $\mu_{A}(x)=\exp \left[-\left(\frac{x-m}{\mathrm{o}}\right)^{2}\right]$. Moreover, the fuzzy set $A$ can be express as $A(m, \sigma)$.

Definition 1: A fuzzy rule base, $R=\bigcup_{j=1}^{N} R_{j}$, is a union of fuzzy rules, in which, each rule $j$ can be expressed as

$$
\begin{aligned}
& R_{j}: \operatorname{IF~} x_{1} \text { is } A_{1 j}\left(m_{1 j}, \sigma_{1 j}\right) \text { and } \ldots \text { and } x_{n} \text { is } A_{n j}\left(m_{n j}, \sigma_{n j},\right. \\
& \operatorname{THEN} u \text { is } B_{j}\left(\theta_{j}, \delta_{j}\right), \quad x_{i} \in X_{i}, u \in U
\end{aligned}
$$

Definition 2: The firing strength of $j$ th rule is

$$
\mu_{R_{1}}(\mathbf{x})=\bigcap_{i=1}^{n} \mu_{A_{i}}\left(x_{i}\right)
$$

Definition 3: A fuzzy rule base, $R$, is said to be complete if and only if there at least exist a rule, $k$, in $R$, such that $\mu_{R_{k}}=0, \forall x_{i} \in X_{i}$.

Definition 4: The fuzzy premise functions, $p(x)$, can be determined by the premise part of $R_{i}$, and can be defined as

$$
p_{j}(\mathbf{x}) \stackrel{\Delta}{\sum_{j=1}^{N} \mu_{R_{j}}(x)}, j=1,2, \ldots, N
$$

Definition 5: The weighted average defuzzification is defined as

$$
u=\frac{\sum_{j=1}^{N} \theta_{j} \mu_{x_{j}}(x)}{\sum_{j=1}^{N} \mu_{z_{j}}(x)} \triangleq \theta^{\mathrm{T}} \mathbf{p}(\mathbf{x})
$$

where $\theta=\left[\theta_{1} \theta_{2} \ldots \theta_{N}\right]^{\mathrm{T}}, \mathbf{p}=\left[p_{1} p_{2} \ldots p_{N}\right]^{\mathrm{T}}$. We have the following fact

Fact: If a fuzzy rule base is complete, then

i). All $p_{j}(x)$ are well-defined, ii). $0 \leq p_{j}(x) \leq 1$.

Therefore, it is important to construct a complete fuzzy rule base in FLC design. We sug- gest two principles to obtain a complete fuzzy rule base: 1) Overlapping all membership functions of every input variable. 2) Take all possible label combinations of input variables.

\section{B. Fuzzy sliding mode control}

In conventional SMC design, the designer must choose a sliding function first, i.e.

$s(\mathbf{x}): R^{n} \rightarrow R$

for the linear case, $s($.$) can be selected as a lin-$ ear combination of state, $s=c^{T} \mathbf{x}$. In this paper, we will adopt the linear sliding function for simplicity. The sliding surface can be viewed as a set of state, in which, the mapping (5) equal to zero, i.e.

$$
S=\left\{\mathbf{x} \mid \mathbf{c}^{\mathrm{T}} \mathbf{x}=0\right\}
$$

Applying the fuzzification operation to fuzzify the crisp sliding surface (set) to a fuzzy one is the main ideal of FSMC. Figure 1 illustrate such concept.

\section{Adaptive algorithm for FSMC}

A class of $\boldsymbol{n}$-th order nonlinear systems considered in this work have the form of

$$
y^{(n)}=f\left(y, \dot{y}, \ldots, y^{(n-1)}\right)+b u, \quad b>0(7)
$$

where $f($.) is a unknown continuous function, $b$ is a unknown positive constant, and $u \in R$ is the system input and $y \in R$ is the system output. To transfer this differential equation to a state space realization, let $r$ be the reference input, and let $\varepsilon=r-y$ be the error signal. Define the state $e_{i}=r^{(i-1)}-y^{(i-1)} \triangleq \varepsilon^{(i-1)}, i=1,2, \ldots, n$, then the system (7) can be represented as the following state equations

$$
\left\{\begin{array}{l}
\dot{e}_{i}=e_{i+1}, \\
\dot{e}_{n}=r^{(n)}-f-b u
\end{array} \quad i=1, \ldots, n-1\right.
$$

Define the sliding function

$$
s=\mathbf{c}^{\mathrm{T}} \mathbf{e}
$$

where $\mathrm{c}=\left[c_{1} c_{2} \ldots, c_{n-1} 1\right]^{\mathrm{T}}$, then when the state on the sliding surface $s=0$, the equivalent control which can keep the state stay on the sliding surface can be derived from setting the derivative of $s, \dot{s}$, equal to zero, that is

$$
u_{e q}=\left.u\right|_{s=0}=b^{-1}\left(-f+r^{(n)}+c^{\mathrm{T}} \mathbf{e}\right)
$$


Moreover, in the sliding mode, $s=0$, the system dynamic can be characterized by the following characteristic polynomial

$$
D^{n-1}+c_{n-1} D^{n-2}+\ldots+c_{1}=0
$$

where $D \stackrel{\Delta}{=} \frac{d}{d}$, with suitable choice of the coefficients $c_{i}$, one can obtain a desired and stable equivalent control system $\dot{\mathbf{e}}=\mathbf{A}_{c} \mathbf{e}$, where $\mathbf{A}_{c} \in R^{n \times n}$, is a companion matrix in controllable canonical form with $-c_{i}$ in the last row and has negative eigenvalues if (11) is Herwitz. To develop adaptive fuzzy sliding mode control law, let's assume that we can find a set of $c_{i}, i=1,2, \ldots, n$, such that $\mathbf{c c}^{\mathrm{T}} \mathbf{A}_{\mathrm{c}}=-\mathbf{Q}$, where $\mathbf{Q}$ is a positive definite matrix.

Without knowing $f($.$) and b$, there is no way to get $u_{e q}$, the main purpose here is to use a fuzzy system to approximate it. Suppose that the adaptive fuzzy sliding mode control law has two parts, one is fuzzy part, $u_{\beta}$, and another one is hitting part, $u_{h}$, i.e.

$$
u=u_{f}+u_{h}
$$

where $u_{f}$ is obtained from a fuzzy rule base described in the Section 2. Now, we derived an adaptive law to adjust the parameter vector $\theta$. Assume there exist a set of parameters $\theta^{*}$ in the universe of discourse $\Theta$, such that $\left(\theta^{*}\right)^{\mathrm{T}} \mathbf{p}$ $u_{e q}$ is minimal. Define a Lyapunov function

$$
V=\frac{1}{2} s^{2}+\frac{b}{2 \eta} \phi^{\mathrm{T}} \phi
$$

where $\phi \stackrel{\Delta}{=} \theta^{*}-\theta$, and $\eta$ is a positive constant. Substitute (12) to the error equation (8), we get

or

$$
\dot{e}_{n}=-\overline{\mathbf{c} e}+b\left(u_{e q}-u_{f}-u_{h}\right)
$$

$$
\dot{\mathbf{e}}=\mathbf{A}_{c} \mathbf{e}+\mathbf{b}_{c}\left(u_{e q}-u_{f}-u_{h}\right)
$$

where $\overline{\mathbf{c}}=\left[\begin{array}{llll}c_{1} & \ldots & c_{n-1} & 0\end{array}\right]^{\mathrm{T}} \mathbf{b}_{\mathrm{c}}=\left[\begin{array}{llll}0 & 0 & \ldots & b\end{array}\right]^{\mathrm{T}} \in R^{n}$. Then

$\dot{V}=-\mathbf{e}^{\mathrm{T}} \mathbf{Q} \mathbf{e}+\frac{b}{\eta} \phi^{\mathrm{T}}(\eta s \mathrm{p}+\dot{\phi})-s b v-s b u_{h}$

where $\mathbf{p}=\mathbf{p}(s)$ is the fuzzy premise function vector described in Section 2, and $v=u_{f}-u_{e q}$. Note that $\dot{\phi}=-\dot{\theta}$, hence, if we choose the adaptive law as

$$
\dot{\boldsymbol{\theta}}=\eta \boldsymbol{\eta}(\mathbf{e}) \mathbf{p}(s)
$$

and $u_{h}$ has sign agree with $s b$ (this part will be derived in the next paragraph), then equation (16) becomes

$$
\dot{V} \leq-\mathbf{e}^{\mathrm{T}} \mathbf{Q} \mathbf{e}-\mathbf{e}^{\mathrm{T}} \mathbf{c} b v
$$

Stability consideration: The purpose of hitting control part in the equation (12) is used to draw the state to hit the sliding surface no matter where the initial state is. To achieve such a goal, let's define a Lyapunov function for $s$

$$
V_{s}=\frac{1}{2} s^{2}
$$

the hitting condition which guarantees the stability of sliding mode control system is

$$
\dot{V}_{s}=s \dot{s}<0
$$

Suppose that we know the upper bound of $f($.) and lower bound of $b$, i.e.

$$
|f| \leq \bar{f} \text {, and } 0<\underline{b} \leq b
$$

Then, we have

$$
\begin{aligned}
\dot{V}_{s} & =s b\left(u_{e q}-u_{f}-u_{h}\right) \\
& \leq|s b|\left(\left|u_{e q}\right|+\left|u_{f}\right|\right)-s b u_{h}
\end{aligned}
$$

To guarantee (22) less then or equal to zero, the hitting control law must be selected as

$u_{h}=\operatorname{sign}(s)\left[\underline{b}^{-1}\left(\bar{f}+\left|r^{(n)}\right|+\left|\overline{\mathbf{c}}^{\mathrm{T}} \mathbf{e}\right|+\left|u_{f}\right|\right)\right]$

Substitute (23) into (22), one can get $\dot{V}_{s} \leq 0$, and the hitting condition can be satisfied, therefore the system is stable.

Practical consideration: In general, the hitting control part described previously is a high gain bang-bang control. It will generate a very large control force and increase the control cost, which is usually undesired. Therefore, we replace the sign function in (23) by a saturation function and modify the control law (12) by

$$
u=u_{f}+\alpha u_{h}
$$

where

$$
\alpha= \begin{cases}1, & |s|>\bar{s} \\ 0, & |s|<\bar{s}\end{cases}
$$

and $\bar{s}$ is a constant specified by the designer.

\section{Simulation results}

In this section, we applied the AFSMC developed in the last section to control an inverted 
pendulum system, see Figure 2, which is highly nonlinear and unstable. The dynamics of the inverted pendulum system can be characterized by two state variables. $x_{1}$ denotes the angle of the pole with respect to the vertical line, and $x_{2}$ denotes the angular velocity of the pole. $\mathrm{Ne}$ glecting the coefficient of friction, the state equation can be expressed as follows

$$
\begin{aligned}
& \dot{x}_{1}=x_{2} \\
& \dot{x}_{2}=\frac{g \sin x_{1}-\cos x_{1}\left(\frac{m l}{m_{c}+m} x_{2}^{2} \sin x_{1}-\frac{1}{m_{c}+m} f\right)}{\frac{4}{3} l-\frac{m l}{m_{c}+m} \cos ^{2} x_{1}}
\end{aligned}
$$

where $g$ is the gravity constant, $9.8 \mathrm{~m} / \mathrm{s}^{2} . m_{c}$ is the mass of the cart, $1.0 \mathrm{~kg}$. $m$ is the mass of the pole, $0.1 \mathrm{~kg} . l$ is the length of the pole, 1 m. $f$ is the input force to the cart. Select $\mathrm{c}=[10$ $1]^{\mathrm{T}}$ and $\eta=10$. Before applying adaptive fuzzy sliding mode control law to the system, the design must define an initial complete rule base, a possible choice is

IF $s$ is $P B(2,0.7) \quad$ THEN $u$ is $Z E(0,2)$

IF $s$ is $P M(1.2,0.3)$ THEN $u$ is $Z E(0,2)$

IF $\mathrm{s}$ is $P S(0.5,0.3) \quad$ THEN $u$ is $Z E(0,2)$

IF $s$ is $Z E(0,0.2) \quad$ THEN $u$ is $Z E(0,2)$

IF $s$ is $N S(-0.5,0.3)$ THEN $u$ is $Z E(0,2)$

IF $\mathrm{s}$ is $N M(-1.2,0.3)$ THEN $u$ is $Z E(0,2)$

IF $s$ is $N B(-2,0.7) \quad$ THEN $u$ is $Z E(0,2)$

Note that consequence part of above rule base are all zero. By applying the adaptive law (17) and the control law (24) with $\alpha=0$, i.e. without using hitting control part, the membership functions of $u$ are shown in Figure 3 at time step $0,50,100$ and 500, respectively. Figure 4 shows the response of the system controlled by such 7-rule AFSMC. By adding the hitting control part to the system, Figure 5 shows an asymptotically sliding mode can be achieved. To view the rule tolerance property of AFSMC, we define another rule base with 5 rules as

IF $\mathrm{s}$ is $P B(2,1)$

$\begin{array}{ll}\text { IF } s \text { is } P S(0.5,0.5) & \text { THEN } u \text { is } Z E(0,2) \\ \text { IF } s \text { is } Z E(0,0.5) & \text { THEN } u \text { is } Z E(0,2)\end{array}$

IF $s$ is $N M(-0.5,0.5)$ THEN $u$ is $Z E(0,2)$

IF $\mathrm{s}$ is $N B(-2,1) \quad$ THEN $u$ is $Z E(0,2)$ the membership functions of $u$ are shown in Figure 6 at time step 0, 50, 100 and 500, respectively. Figure 7 shows the response of the system controlled by such 5-rule AFSMC. Compare Figure 4 with Figure 7, we see that even the initial rule bases are so different, the AFSMC can yield similar responses, such a rule tolerance property can reduce the expertise dependency in the designing procedure.

\section{Conclusions}

In this paper, we developed an adaptive fuzzy sliding mode controller, which has the following characteristics

1) The membership functions of consequence can be tuned automatically.

2) Does not require the mathematical model of the system.

3) Expertise dependency can be reduced by the rule tolerance property.

4) By applying the hitting control part, the stability of control system can be guaranteed.

5) Dynamic behavior of the control system can be specified by an user-defined sliding surface.

\section{Reference}

[1] C. C. Lee, "Fuzzy logic in control systems: Fuzzy logic controller, parts I and II," IEEE Trans. Syst., Man, Cybern., vol. 20, no. 2, pp. 404-435, 1990.

[2] E. M. Mamdani, "Application of fuzzy algorithms for control of simple dynamic plant," Proc. IEE, vol. 121 , no. 12 , pp. 1585-1588, 1974

[3] M. Sugeno and M. Nishida, "Fuzzy control of model car," Fuzzy Sets and Systems, vol. 16, pp. 103-113, 1985.

[4] S. C. Lin and C. C. Kung, "A linguistic fuzzy sliding mode controller," Proc. of 1992 A.C.C., pp. 1904-1905.

[5] V. I. Utkin, Sliding modes and their application in variable structure system, Moscow: Mir, 1978 (English translation). 


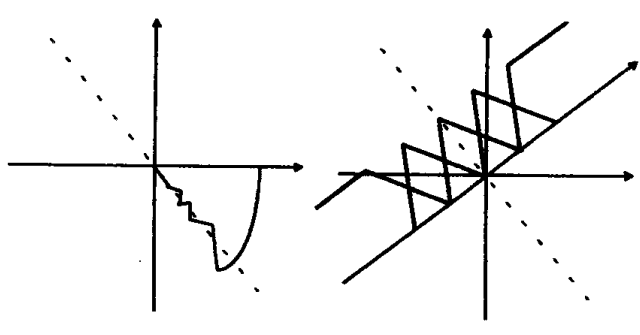

Figure 1 Fuzify the crisp sliding surface

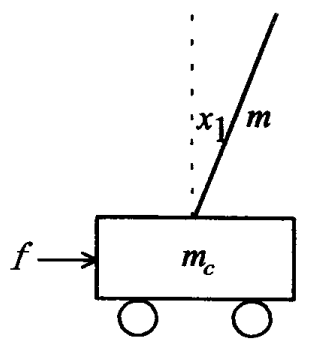

Figure 2 The inverted pendulum system
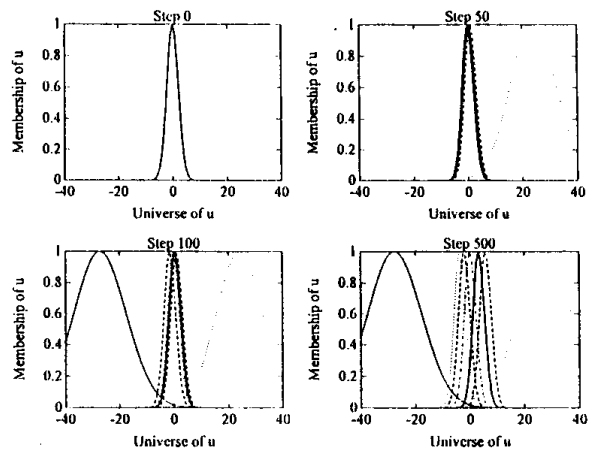

Figure 3 The membership functions of $u$ in the 7-rule AFSMC under adjusting.

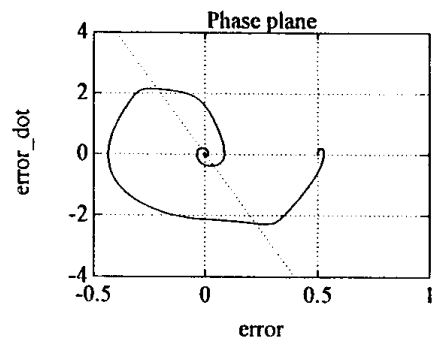

Figure 4 The response of 7-rule AFSMC

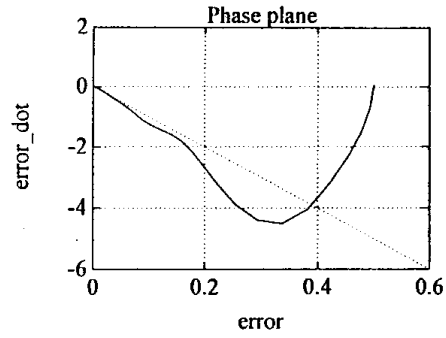

Figure 5 The response with hitting control
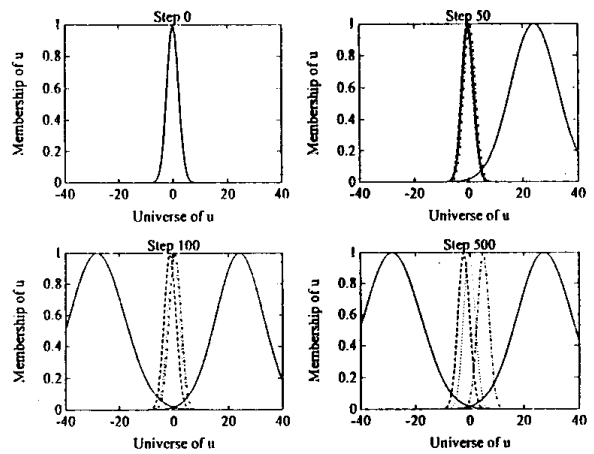

Figure 6 The membership functions of $u$ in the 5-rule AFSMC under adjusting.

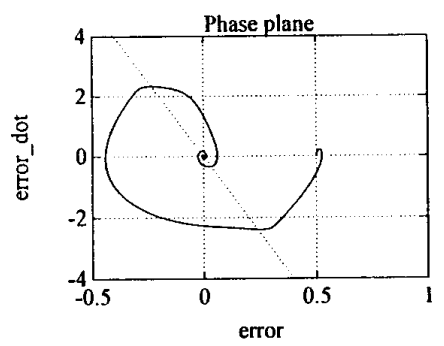

Figure 7 The response of 5-rule AFSMC 